\title{
Stromal CD10 expression in invasive breast carcinoma
}

\begin{tabular}{llll}
\hline Haval D. Ali * & Jalal A. Jalal $* *$ & Ava T. Ismail $* * *$ & Wahda MT. Alnuaimy $* * * *$ \\
\hline & Abstract
\end{tabular}

Background and Objective: This study aimed to assess the immunohistochemical expression of stromal CD10 in invasive breast carcinoma and determine its relationship with some clinicopathological parameters.

Methods: A cross-sectional study was conducted on 91 female breast cancer cases collected from the archive of pathology department in Rizgary Teaching Hospital and some private labs during the period from January 2012 to August 2015. CD10 expression was detected by immunohistochemistry using labeled polymer and enhanced polymer systems (Dako EnVision ${ }^{\mathrm{TM}}$ Flex) Dakoprotocol. The scoring was based on the intensity and percentage of the stained stromal cells. Then CD10 immunoexpression was correlated with the age of patients, tumor size, histopathological types, tumor grade, lymphovascular invasion, lymph node status, and tumor stage.

Results: Stromal CD10 expression was observed in $70.4 \%$ of the cases. A statistically significant positive correlation was seen between stromal CD10 and the tumor subtype $(P=0.02)$, tumor grade $(P=0.025)$, lymphovascular invasion $(P=0.002)$, and lymph node status $(P=0.047)$, however, no statistically significant association was identified between stromal CD10 expression and age, tumor size and tumor stage.

Conclusion: Stromal CD10 is expressed in the majority of cases of invasive breast carcinoma and concerning the positive correlations between stromal CD10 expression and tumor grade, lymphovascular invasion, and lymph node status suggest a strong effect of stromal CD10 expression on aggressive behavior of breast carcinoma.

Keywords: Invasive breast carcinoma; Stroma; CD10; Immunohistochemistry; Histopathology.

\section{Introduction}

Carcinoma of the breast is a highly heterogeneous tumor, which only happens to originate in the same anatomical site. Breast tumors of the similar histological type can show remarkably different clinical behavior, response to therapy and prognosis. $^{1-6} \mathrm{~A}$ better understanding of stromal contribution to cancer progression will identify specific signals that promote growth, dedifferentiation, invasion and ectopic survival of tumor cells and may eventually result in the identification of new therapeutic targets for future treatment. Moreover, stromal markers are now emerging as novel markers in assessing the prognosis of invasive breast cancer, CD10 is a 90-110 kd cell surface zinc dependent metalloproteinase. Since CD10 is structurally similar to matrix metalloproteinase and stromelysin, it might facilitate cancer cell invasion and/or metastasis. CD10 related DNA sequences have been found on human chromosome 3 , at 3q21-27. Stromal CD10 expression is associated with more aggressive behavior in various epithelial malignancies. ${ }^{7,8}$ In gastric carcinoma, CD10 positive stromal cells are correlated with vascular invasion and metastasis. ${ }^{9}$ In nasopharyngeal

* Department of Histopathology, Rizgary Teaching Hospital, Erbil, Iraq.

** Department of Pathology, College of Medicine, Hawler Medical University, Erbil, Iraq.

*** Department of Clinical Analysis, College of Pharmacy, Hawler Medical University, Erbil, Iraq.

$* * * *$ Department of Pathology, College of Medicine, University of Mosul, Mosul, Iraq. 
carcinoma stromal CD10 expression correlated with tumor progression. ${ }^{10}$ The interaction of CD10 positive stromal cells with breast carcinoma cells might induce the carcinoma cells to proceed through the cell cycle and activate their movement. Many studies have addressed the clinical significance of stromal CD10 expression in invasive breast carcinoma. ${ }^{7,8}$ These studies have indicated an association between stromal CD10 expression and poor prognosis. To our knowledge, no studies have been done in our region to clarify this issue; this led us to conduct this study. This study aimed to assess the immunohistochemical expression of stromal CD10 in invasive breast carcinoma and to determine its relationship with some clinicopathological parameters.

\section{Methods}

This is a cross-sectional study was conducted after permission approval granted by Erbil ministry of health office. Samples of primary breast carcinoma, obtained from 91 female patients underwent radical mastectomy, quadrantectomy, lumpectomy with or without axillary clearance or true cut biopsies for breast cancer. They were collected from Rizgary Teaching Hospital and some private labs in Erbil and Duhok cities. Tissue blocks were collected during a period spanning from January 2012 to August 2015. For each case, all Hematoxyline and Eosin stained sections were reviewed concerning the type and the grade of the tumor, the lymphovascular invasion, the axillary lymph node status and tumor stage. The information was gained from the patient's case sheaths from the histopathology department archive. The tumors were typed according to the WHO classification system and graded according to the Nottingham modification of the Bloom - Richardson grading scheme. ${ }^{11} \mathrm{~A}$ full clinicopathological staging was evaluated, depending on American Joint Committee on Cancer (AJCC) criteria. $^{12}$

\section{Immunohistochemical method}

Labeled polymer and enhanced polymer systems (Dako EnVision ${ }^{\mathrm{TM}}$ Flex) method according to Dako recommendation was used to stain the tissue with the CD10 antibody. The staining steps and incubation times are pre-programmed into the software of Dako autostainer link 48, where substrate Buffer, Envision TM FLEX Peroxidase-Blocking Reagent (as endogenous enzyme block), FLEX Monoclonal Mouse Anti-Human CD10 Clone 56C6 Ready-to-Use (as primary antibody), Envision TM FLEX+ Mouse linker (as a secondary reagent), EnVision/ HRP (as a labeled polymer), DAB+ Chromogen (as substrate Chromogen), Envision TM FLEX hematoxylin (as a counter stain) and distilled water were applied on the slides. Then slides removed from the autostainer link 48 and put in graded ethanol $(70 \%, 100 \%, 100 \%, 2 \times 2 \times 2$ minutes) respectively, then in xylene (2 minutes). The slides were mounted in Canada Balsam and examined under light microscope. Positive and negative control slides were involved in each run of staining. Moreover, the normal myoepithelial cells lining the acinar and ductal structures in normal parenchyma adjacent to the tumor were used as positive internal controls too. While negative control slides were prepared from the same tissue block, they incubated with TBS instead of the primary antibody.

\section{Evaluation of CD10 Immunostaining}

The scoring system used in this study was based on the study of Makretsov et al. ${ }^{13}$ which divided the cases into three groups named as negative, weakly positive, and strongly positive. Every section was carefully examined at power magnification (x100) for the presence of tumor stromal immunostaining. The staining of the cell membrane of the stromal cells was semi quantitatively scored as:

Negative (no staining).

Weak positive (either diffuse weak staining or weak or strong focal staining in less than $30 \%$ of stromal cells per slide). 
Strong positive (defined as strong staining of $30 \%$ or more of the stromal cells per slide).

\section{Statistical analysis}

The collected data was analyzed using the statistical package for the social sciences (version 22) (SPSS Inc., Chicago, USA). Association between stromal CD10 expression and clinicopathological factors were evaluated using the Chi square test, and a $P$ value equal or less than
0.05 was considered significant.

\section{Results}

Ninety one cases of invasive breast carcinoma were included in this study. Age of the patients ranged from 23-86 years with a mean age of $50.62 \pm 13.48$ years. The size of the tumors ranged from 1-10 $\mathrm{cm}$ with a mean size of $3.87 \pm 1.87 \mathrm{~cm}$. The other pathological data of the sampled patients are summarized in Table1.

Table 1: The pathological data of the sampled cases.

\begin{tabular}{|c|c|c|c|}
\hline Variables & Categories & No. & $\%$ \\
\hline \multirow[t]{6}{*}{ Tumor sub-types } & NST & 83 & 91.2 \\
\hline & Invasive lobular & 2 & 2.2 \\
\hline & Combined & 2 & 2.2 \\
\hline & Mucinous & 2 & 2.2 \\
\hline & Medullary & 1 & 1.1 \\
\hline & Papillary & 1 & 1.1 \\
\hline \multirow{3}{*}{$\begin{array}{l}\text { Tumor grade (invasive } \\
\text { breast carcinoma, NST) }\end{array}$} & Grade I & 1 & 1.2 \\
\hline & Grade II & 45 & 54.2 \\
\hline & Grade III & 37 & 44.6 \\
\hline \multirow[t]{2}{*}{ Lymphovascular invasion } & Present & 51 & 56 \\
\hline & Absent & 40 & 44 \\
\hline \multirow[t]{5}{*}{ Lymph node involvement } & Zero & 22 & 24.2 \\
\hline & $1-3$ & 17 & 18.7 \\
\hline & $4-9$ & 11 & 12.1 \\
\hline & $\geq 10$ & 17 & 18.7 \\
\hline & No data available & 24 & 26.4 \\
\hline \multirow[t]{7}{*}{ Tumor stage } & IA & 5 & 5.5 \\
\hline & $\| \mathrm{A}$ & 15 & 16.5 \\
\hline & IIB & 16 & 17.6 \\
\hline & IIIA & 13 & 14.3 \\
\hline & IIIB & 1 & 1.1 \\
\hline & IIIC & 17 & 18.7 \\
\hline & No data available: & 24 & 26.4 \\
\hline
\end{tabular}


Majority of the sampled cases were mastectomy specimens with lymph node dissection 48 (52.7\%), followed by lumpectomy 27 cases $(29.7 \%)$, five cases out of them with lymph node dissection. Fourteen cases (15.4\%) were quadrantectomy with lymph node dissection and two cases $(2.2 \%)$ were true cut biopsies. Immunostaining was performed on all 91 cases. No stromal expression of CD10 was detected in the normal breast, although the non-neoplastic myoepithelial cells in normal parenchyma adjacent to the tumor, used as a built-in positive control in this study, constantly expressed CD10. There was no expression of CD10 in ductal cells, fibroblasts, and adipose cells. CD10 was found to be positive in $70.4 \%$ (64 cases), out of which $46.2 \%$ (42cases)showed weak immunoreactivity, as shown in Figure 1 and $24.2 \%$ (22 cases) showed strong immunoreactivity, as shown in Figure 2, while $29.7 \%$ (27 cases) were negative.

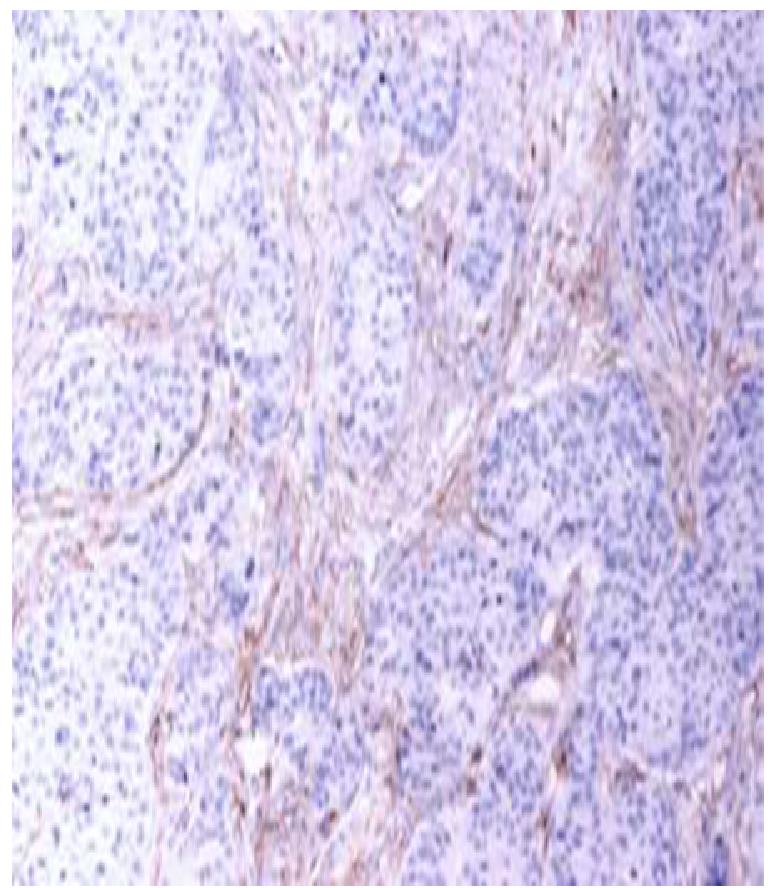

Figure 1: Invasive ductal carcinoma (NST) with weak CD10 stromal immunostaining (IP- Mayer's Hematoxylin counter stain $\mathrm{x} 100)$

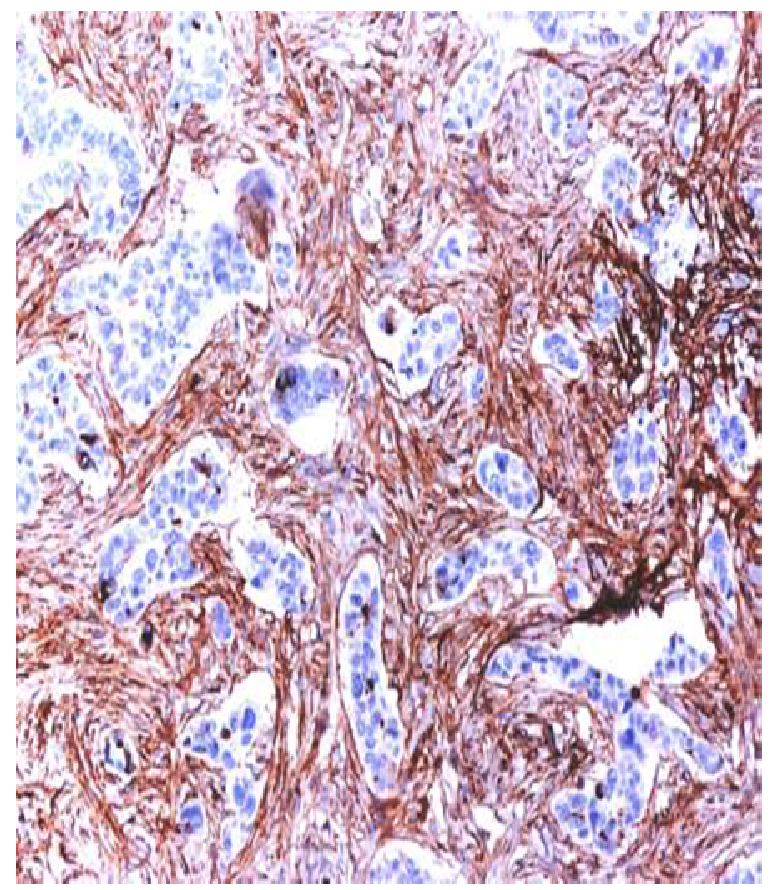

Figure 2: Invasive ductal carcinoma(NST) with strong CD10 stromal immunostaining (IP- Mayer's Hematoxylin counter stain $\mathrm{x} 100)$ 
The correlations between stromal CD10 expression and clinicopathological characteristics of patients are illustrated in Table 2. A statistically significant association was seen between stromal CD10 expression and the tumor subtype; especially infiltrative ductal carcinoma, tumor grade, in which most of the strong positive CD10 cases were Grade III; lymphovascular invasion; a higher percent in CD10 positivity was seen in cases with lymphovascular invasion than those without invasion; and lymph node status; cases with $\mathrm{N} 1$ followed by N3 group showed the highest frequency for CD10 immunostaining. No statistically significant association was identified between stromal CD10 expression and age, Although the highest frequency of CD10 positivity was observed at 40-60 years age group; tumor size; despite the fact that all $\mathrm{T}$ categories showed positivity for CD10, and tumor stage; Although most of the stromal CD10 positive cases were stage IIIC and IIB.

Table 2: The correlation between CD10 expression and clinicopathological parameters.

\begin{tabular}{|c|c|c|c|c|c|c|}
\hline \multirow{2}{*}{ Variables } & \multirow{2}{*}{ Categories } & \multirow{2}{*}{ No. } & \multicolumn{3}{|c|}{ CD 10 expression } & \multirow{2}{*}{$P$ value } \\
\hline & & & Negative & Weak & Strong & \\
\hline \multirow[t]{3}{*}{ Age groups } & $<40$ & 16 & $4(25)$ & $9(56)$ & $3(18)$ & \multirow{3}{*}{0.3} \\
\hline & $40-60$ & 51 & $14(27.5)$ & $22(43.1)$ & $15(29.4)$ & \\
\hline & $>60$ & 24 & $10(41.7)$ & 9 (37.5) & $5(20.8)$ & \\
\hline \multirow[t]{4}{*}{ Tumor size } & $\mathrm{T} 1$ & 17 & $5(29.4)$ & $8(47.1)$ & $4(23.5)$ & \multirow{4}{*}{0.5} \\
\hline & $\mathrm{T} 2$ & 48 & $12(25)$ & $21(43.8)$ & 15(31.3) & \\
\hline & T3 & 21 & $9(42.9)$ & $10(47.6)$ & $2(9.5)$ & \\
\hline & $\mathrm{T} 4$ & 5 & $1(20)$ & $3(60)$ & $1(20)$ & \\
\hline \multirow[t]{6}{*}{ Histological type } & Ductal & 83 & $21(25.3)$ & $41(49.4)$ & $21(25.3)$ & \multirow{6}{*}{0.020} \\
\hline & Lobular & 2 & $2(100)$ & $0(0)$ & $0(0)$ & \\
\hline & Combined & 2 & $1(50)$ & $0(0)$ & $1(50)$ & \\
\hline & Mucinous & 2 & $2(100)$ & $0(0)$ & $0(0)$ & \\
\hline & Medullary & 1 & $0(0)$ & $1(100)$ & $0(0)$ & \\
\hline & Papillary & 1 & $1(100)$ & $0(0)$ & $0(0)$ & \\
\hline \multirow[t]{3}{*}{ Tumor grade } & Grade I & 3 & $3(100)$ & $0(0)$ & $0(0)$ & \multirow{3}{*}{0.025} \\
\hline & Grade II & 49 & $13(26.5)$ & $27(55.1)$ & $9(18.4)$ & \\
\hline & Grade III & 39 & $11(28.2)$ & $15(38.5)$ & $13(33.3)$ & \\
\hline \multirow{2}{*}{$\begin{array}{l}\text { Lymphovascular } \\
\text { invasion }\end{array}$} & Present & 51 & $14(27.5)$ & $24(47.1)$ & $13(25.5)$ & \multirow[b]{2}{*}{0.002} \\
\hline & Absent & 40 & $13(32.5)$ & $18(45)$ & $9(22.5)$ & \\
\hline \multirow{4}{*}{$\begin{array}{l}\text { Lymph node } \\
\text { status }\end{array}$} & No & 22 & $10(45.5)$ & $6(27.3)$ & $6(27.3)$ & \multirow{4}{*}{0.047} \\
\hline & N 1 & 17 & $1(5.9)$ & $11(64.7)$ & $5(29.4)$ & \\
\hline & $\mathrm{N} 2$ & 11 & $4(36.4)$ & $4(36.4)$ & $3(27.3)$ & \\
\hline & N 3 & 17 & $4(23.5)$ & $10(58.8)$ & $3(17.6)$ & \\
\hline \multirow[t]{6}{*}{ Tumor stage } & IA & 5 & $3(60)$ & $0(0)$ & $2(40)$ & \multirow{6}{*}{0.6} \\
\hline & $\| A$ & 15 & $4(26.7)$ & $7(46.7)$ & $4(26.7)$ & \\
\hline & IIB & 16 & 3 (18.8) & $8(50)$ & $5(31.3)$ & \\
\hline & IIIA & 13 & $5(38.5)$ & $5(38.5)$ & $3(23.1)$ & \\
\hline & IIIIB & 1 & $0(0)$ & $1(100)$ & $0(0)$ & \\
\hline & IIIC & 17 & $4(23.5)$ & $10(58.8)$ & $3(17.6)$ & \\
\hline
\end{tabular}




\section{Discussion}

The immunohistochemical evaluation of the stromal CD 10 expression in tumor tissue is an important parameter in the assessment of the prognosis and management of invasive carcinoma of the breast. Moreover, it has been reported that carcinoma of the breast that not expressed stromal CD 10 behave better, both clinically and biologically than invasive breast carcinoma that expressed it. ${ }^{7,8,13-21}$ In the present study, stromal CD10 expression was found in $70.4 \%$ of the cases (including $46.2 \%$ weakly positive and $24.2 \%$ strongly positive specimens) which is close to the frequency observed by other studies, while higher than others as seen below. Makretsov et al. $^{13}$ found stromal CD10 expression in $79 \%$ of invasive breast carcinomas. Using the same scoring system by Makretsov et al., Hosni et al. ${ }^{14}$ revealed strong stromal CD10 immunostaining in $74 \%$ of invasive duct carcinoma and Mohammadizadeh et al. ${ }^{15}$ study showed $81.6 \%$ of the cases to be stromal CD10 positive. While $64 \%$ of cases were CD10 positive in a study by Taghizadeh-Kermani et al. ${ }^{16}$ Masaki et al. ${ }^{17}$ judged the expression of CD10 to be positive when more than $10 \%$ of the stromal cells around the neoplastic epithelial cells were positive. Based on this criterion, they detected stromal CD10 expression in $19 \%$ of invasive ductal carcinomas. Iwaya et al. ${ }^{18}$ used the same criterion as Masaki et al. to define the stromal CD10 expression and found its expression in $18 \%$ of invasive ductal carcinomas. In a study by Puri et al. ${ }^{19}$ stromal CD10 expression was considered as negative when less than $10 \%$ of the stromal cells were immunoreactive with the marker. Cases with $10-30 \%$ positive stromal cells were considered as weakly positive and the presence of more than $30 \%$ positive stromal cells was defined as strongly positive. According to this definition, stromal CD10 expression was detected in $80 \%$ of the cases out of which $40 \%$ were weakly positive and $60 \%$ were strongly positive. Sadaka et al. ${ }^{20}$ considered CD10 positive for specimens with more than $10 \%$ of the tumor stromal cells, strong positive CD10 when there is strong staining more than $30 \%$ of tumor stromal cells and they found that $28.6 \%$ of the case were positive for CD10. This wide range of detection of the stromal CD10 expression in different studies may be attributed to the number of studied cases, the immunohistochemical methodology used including tissue fixation, the choice of the antibody, the sensitivity of the detection system and the determination of the criteria used for the positive result. Also, differences in the population group, diversity of risk habits and variation of genetic predisposition may contribute to that wide range of stromal CD10 expression that reported in different countries. ${ }^{13-23}$ Similar to this study, Makretsov et al., ${ }^{13}$ Iwaya et al., ${ }^{18}$ Masaki et al., ${ }^{17}$ Puri et al., ${ }^{19}$ Sadaka et al. ${ }^{20}$ and Hosni et al. $^{14}$ failed to show a significant correlation between between tumor size and CD10 expression. While Kim et al. ${ }^{21}$ Mohammadizadeh et al. ${ }^{15}$ and Taghizadeh-Kermani et al. ${ }^{16}$ have found the opposite. The lack of compatibility between the studies regarding tumor size because each study used a different method to categorize the tumor size into two different groups and the study that showed a wide range between these two categories reported a statistically significant relation between stromal CD10 expression and the tumor size. The present study and Mohammadizadeh et al. ${ }^{15}$ revealed a significant relationship between stromal CD10 expression and tumor subtype which is contrary to what had been reported by Makretsov et al. ${ }^{13}$ The majority of this study cases fitted histologically into grade II, but most of strong positive stromal CD10 expression were grade III. Makrestov et al. ${ }^{13}$ Mohammadizadehet al. ${ }^{15}$ and Hosni et al. ${ }^{14}$ reported similar findings. The present study and the study done by Makretsov et al., ${ }^{13}$ $\mathrm{Kim}$ et al.," Mohammadizadeh et al., ${ }^{21}$ 
Taghizadeh-Kermani et al., ${ }^{16}$ Sadaka et al., ${ }^{20}$ and Hosni et al. ${ }^{14}$ showed a statistically significant positive correlation between stromal CD10 expression and tumor grade. The contradictory was found by Iwaya et al. ${ }^{18}$ and Puri et al. ${ }^{19}$ Concerning lymphovascular invasion, similar to Sadaka et al. ${ }^{20}$ this study was able to report a highly significant association between stromal CD10 expression and lymphovascular invasion. As far as the lymph node involvement concerned, the results of this study agree with studies done by Iwaya et al., ${ }^{18}$ Masaki et al., ${ }^{17}$ Mohammadizadeh et al., ${ }^{15}$ Taghizadeh-Kermani et al., ${ }^{16}$ Sadaka et al. $^{20}$ and Kim et al. ${ }^{21}$ who demonstrate stromal CD10expression to be significantly correlated with nodal involvement. However, Makretsov et al. ${ }^{13}$ and Hosni et al. ${ }^{14}$ failed to establish a positive correlation between stromal CD10 expression and lymph node status. Probably the negative node cases were strikingly outnumbered the positive nodes cases in Makretsov et al. study and the very small sample size in Hosni et al. study are the reasons that brought them to statistically insignificant correlations. Iwaya et al. ${ }^{18}$ concluded that stromal CD10 expression has no significant association with the cancer stage; the same result was identified in the present study. The reason behind such non -significant results is probably that Iwaya et al. did not include in their study cases with stage IIIB, IIIC and IV. This study also lacked stage IV and IB cases. Furthermore, only one case with tumor stage IIIB was included. The survival data of the patients were unavailable in this study. In a nutshell, the results of this study in accordance with those by other researchers draw a new perspective of therapeutic strategies in future. ${ }^{22,23}$ Treatments targeted to reduce the effect of the CD10 positive stromal component on aggressive behavior of breast cancer may be promising in this regard.

\section{Conclusion}

Stromal CD10 is expressed in $70.4 \%$ of cases of invasive breast carcinoma and the highest frequency of strong positivity of CD10 immunostaining was seen in Grade III tumors and tumors with lymphovascular invasion. Also, stromal CD10 expression was significantly associated with the histological type, tumor grade, lymphovascular invasion, and lymph node metastasis, however, no statistically significant association was identified between stromal CD10 expression and age, tumor size and tumor stage.

\section{Competing interests}

The authors declare that they have no competing interests.

\section{References}

1. Torre LA, Bray F, Siegel RL, Ferlay J, Lortet-Tieulent J, Jemal A. Global cancer statistics 2012. Can J Clin 2015; 65:87-108.

2. Howlader N, Noone AM, Krapcho M, Miller D, Bishop K, Altekruse SF, et al. SEER Cancer Statistics Review, 1975-2013, National Cancer Institute. Bethesda, MD, http://seer cancer gov/ csr/1975 2013/, based on November 2015 SEER data submission, posted to the SEER web site, April 2016.

3. OECD/European Union 2010: Screening, Survival and Mortality for Breast Cancer, in Health at a Glance: Europe; 2010. OECD Publishing.

4. American Cancer Society. Breast Cancer Facts \& Figures 2015-2016. Atlanta: American Cancer Society, Inc.; 2015. P. 23-6.

5. Weigel MT, Dowsett M. Current and emerging biomarkers in breast cancer: prognosis and prediction. Endocr Relat Can 2010; 17:R245-62.

6. Badve S, Dabbs DJ, Schnitt SJ, Baehner FL, Decker T, Eusebi V, et al. Basallikeand triple-negative breast cancers: a critical review with an emphasis on the implications for pathologists and oncologists. Mod Pathol 2011; 24:157-67.

7. De Wever O, Mareel M. Role of tissue stroma in cancer cell invasion. J Pathol 2003; 200:429-47.

8. Jana SH, Jha BM, Patel C, Jana D, Agarwal A. CD10-a new prognostic stromal marker in breast carcinoma, its utility, limitations and role in breast cancer pathogenesis. Ind J Pathol Microbio 2014; 57:530-6.

9. Huang WB, Zhou XJ, Chen JY, Zhang LH, Meng K, Ma HH, et al. CD10-positive stromal cells in gastric carcinoma: correlation with invasion and metastasis. JPN J Clin Oncol 2005; 35:245-50. 
10. Braham H, Trimeche M, Ziadi S, Mestiri S, Mokni $\mathrm{M}$, Amara $\mathrm{K}$, et al. CD10expression by fusiform stromal cells in nasopharyngeal carcinoma correlates with tumor progression. Virch Arch 2006; 449:220-4.

11. Rakha EA, El-Sayed ME, Lee AH, Elston CW, Grainge MJ, Hodi Z, et al. Prognostic significance of Nottingham histological grade in invasive breast carcinoma. J Clin Oncol 2008; 26:3153-8.

12. Singletary SE, Allred C, Ashley P, Bassett LW, Berry $\mathrm{D}$, Bland $\mathrm{KI}$, et al. Staging system for breast cancer: revisions for the 6th edition of the AJCC Cancer Staging Manual. Surg Clin North Am 2003; 83:803-19.

13. Makretsov NA, Hayes M, Carter BA, Dabiri S, Gilks CB, Huntsman DG. Stromal CD10 expression in invasive breast carcinoma correlates with poor prognosis, estrogen receptor negativity, and high grade. Mod Pathol 2007; 20: 84-9.

14. Hosni HN, Abd El-Aziz A, Tabak SA, Elsayed M. Immunohistochemical Study of Stromal CD10 Expression in Mammary Duct Carcinoma. Med J Cairo Univ 2012;80:37-44.

15. Mohammadizadeh F, Salavati M, Afshar moghaddam N. CD10 expression in stromal component of invasive breast carcinoma: A potential prognostic determinant. J Res Med Sci 2012; 17:S194-9.

16. Taghizadeh-Kermani $A$, Jafarian $A H$, Ashabyamin R, Seilanian-Toosi M,Pourali L, Asadi $M$, et al. The Stromal Overexpression of CD10 in Invasive Breast Cancer and its Association with Clincophathologic Factors. Iran J Can Prev 2014; 7:17-21.

17. Masaki T, Keiichi I, Masahiko K, Miki I. The stromal expression of CD10 in breast carcinoma. $\mathrm{J}$ of Tokyo Med Uni 2001; 59:45-50.

18. Iwaya K, Ogawa H, Izumi M, Kuroda M, Mukai K. Stromal expression of CD10 in invasive breast carcinoma: a new predictor of clinical outcome. Virch Arch 2002; 440:589-93.

19. Puri V, Jain M, Thomas S. Stromal Expression of CD10 in Invasive Breast Carcinoma and Its Correlation with ER, PR, HER2-neu, and Ki67. Int J Bre Can 2011; 2011:1-4.

20. Sadaka E, Almorsy W, Elsaka A. CD10 Expression as a Prognostic Factor in Female Patients with Invasive Ductal Carcinoma of the Breast. J Ame Sci 2016; 12:71-7.

21. Kim HS, Kim GY, Kim YW, Park YK, Song JY, Lim SJ. Stromal CD10 expression and relationship to the E-cadherin/beta-catenin complex in breast carcinoma. Histopathol 2010; 56:708-19.

22. Thomas S, Babu RJ, Agarwal K, Puri V, Jain $M$, Andley $M$, et al. Effect of neoadjuvant chemotherapy on stromal CD10 antigens in breast cancer - a preliminarystudy. Ind J Can 2013; $50: 46-51$.
23. Desmedt C, Majjaj S, Kheddoumi N, Singhal SK, Haibe-Kains B, El OuriaghliF, et al. Characterization and clinical evaluation of CD10+ stroma cells in the breast cancer microenvironment. Clin Can Res 2012; 18:100414. 